\title{
Temporal expectation driven by rhythmic cues compared to that driven by symbolic cues provides a more precise attentional focus in time
}

\author{
Zhihan $\mathrm{Xu}^{1,2}$ - Yanna Ren ${ }^{2,3} \cdot$ Ting Guo $^{2} \cdot$ Aijun Wang ${ }^{4,5} \cdot$ Takanori Nakao $^{2}$ - Yoshimichi Ejima ${ }^{2}$. Jiajia Yang ${ }^{2}$. \\ Satoshi Takahashi ${ }^{2}$. Jinglong $\mathrm{Wu}^{2,6} \cdot$ Qiong $\mathrm{Wu}^{2,7} \cdot$ Ming Zhang ${ }^{4,5,7}$
}

Accepted: 21 September 2020 / Published online: 23 October 2020

(C) The Psychonomic Society, Inc. 2020

\begin{abstract}
Temporal expectation is the ability to select the precise point in time for doing something to produce an optimal effect. Two sources of information that humans use to generate temporal expectations are rhythmic and symbolic cues. Both types of cues have been proven effective in directing attention to a future point in time resulting in improved performance. However, the temporal precision of the two forms of temporal expectation have rarely been compared. In the current study, 17 participants performed two temporal expectation tasks in which either a rhythmic cue or a symbolic cue indicated that a future target would appear after a 500-ms (short) or 1,500-ms (long) interval; the target appeared at the expected time in 54\% of trials and at an unexpected earlier or later interval in $36 \%$ of trials. In both tasks, we observed that the reaction time (RT) curves were U-shaped, with a slower RT for the earlier and later unexpected intervals and a faster RT for intervals approaching the expected point in time. Furthermore, we found a significant interaction between task and the quadratic term of temporal expectation, which indicates that the U-shaped RT curves for the rhythmic cue task are steeper than those for the symbolic cue task. Thus, the current results revealed that compared with symbolic cues, temporal expectation driven by rhythmic cues provides a more precise attentional focus in time.
\end{abstract}

Keywords Rhythm $\cdot$ Symbolic $\cdot$ Temporal expectation $\cdot$ U-shaped curve $\cdot$ Temporal window

\section{Introduction}

Temporal expectation refers to the process of focusing attention on a specific point in time to optimize behaviour, which is a fundamental survival ability in our daily lives. Humans rely on two sources of information to generate temporal expectations: rhythmic cues, if available, and symbolic cues.

Zhihan Xu and Yanna Ren contributed equally to this work and should be considered co-first authors.

Qiong $\mathrm{Wu}$

wuqiong@usts.edu.cn

Ming Zhang

psychzm@163.com

1 Department of Foreign Language, Ningbo University of Technology, Ningbo 315211, Zhejiang, China

2 Cognitive Neuroscience Laboratory, Graduate School of Natural Science and Technology, Okayama University, Okayama 7008530, Japan
Rhythmically defined temporal expectation has been suggested to be a stimulus-driven orientation of attention in time (Breska \& Deouell, 2014; Rohenkohl, Coull, \& Nobre, 2011). When participants experience a non-random temporal structure, they can spontaneously adapt to the temporal regularity and build a temporal pattern of the fixed interval, which can be used to predict the likely point in time when the next event 
will occur in order to optimize perceptual or motor performance (Barnes \& Jones, 2000; Jones \& Boltz, 1989; Large \& Jones, 1999). Common findings across studies of temporal expectation driven by rhythmic cues are that, by matching the rhythm's pace, better detection and discrimination occur when an event appears at the expected interval rather than early or late (Doherty, Rao, Mesulam, \& Nobre, 2005; Jones, Moynihan, MacKenzie, \& Puente, 2002; McAuley \& Jones, 2003; Sanabria, Capizzi, \& Correa, 2011).

Conversely, temporal expectation driven by symbolic cues (such as the presentation of a digit or a coloured circle) directs attention through a goal-directed voluntary mechanism (Capizzi, Sanabria, \& Correa, 2012). Depending on the mnemonic representations of the learned cue-target interval, the symbolic, informative cue informs participants whether an upcoming target would likely appear after a short or long delay. Research on temporal expectations triggered by symbolic cues has revealed that compared to misleading (invalid) and non-informative (neutral) cues, valid symbolic cue presentation results in faster and more accurate performance (Correa, Lupianez, Milliken, \& Tudela, 2004; Coull, Frith, Buchel, \& Nobre, 2000; Coull \& Nobre, 1998).

Temporal expectations driven by both rhythmic and symbolic cues have been proven effective in directing attention to a particular point in time for improving performance. However, whether the attentional precision of the two forms of temporal expectation is the same remains unexplored. However, Trivino et al. (2011) have directly compared the two forms of temporal expectation in which a rhythmic cue task and a symbolic cue task were administered to a frontal brain lesion group and a control group. In the rhythmic cue task, the predictive temporal information regarding the target onset time was provided by a fast rhythm or a slow rhythm. In the symbolic cue task, the predictive information was provided by a symbolic cue (short line or long line). In their studies, Trivino et al. did not find a significant difference in the speed-up reaction time (RT) between the two tasks in the control group, even though it seemed a difference could be seen in reducing false alarms between the two tasks from their results (as they used a go/no-go task, in which behavioural performance could be improved through both speeding up RTs and reducing false alarms); however, because they did not perform a statistical analysis of the false alarms, the behavioural differences between the two types of temporal expectation require further investigation (Trivino, Arnedo, Lupianez, Chirivella, \& Correa, 2011). Moreover, Correa et al. (2014) utilized similar tasks and showed that transcranial magnetic stimulation (TMS) on either left or right frontal sites (vs. sham) increased the temporal orienting effect in the symbolic cue task, whereas frontal TMS did not influence performance in the rhythmic cue task. There appeared to be a greater cueing effect (i.e., the difference between the RT for invalid and valid cues) for the rhythmic cue task than for the symbolic cue task under the sham TMS condition. Nonetheless, it is not possible to draw conclusions because of the lack of a statistical comparison (Correa, Cona, Arbula, Vallesi, \& Bisiacchi, 2014). Thus, in the current study, we adapted the two temporal expectation tasks and used a detection task to compare the two forms of temporal expectation directly.

\section{Methods}

\section{Participants}

A total of 17 right-handed volunteer participants (15 males and two females) with a mean age of 23.3 years (range 2128) from Okayama University participated in the study. The participants had normal or corrected-to-normal vision and no record of psychiatric disorders. The study was approved by the institutional ethics committee, and all participants gave written informed consent before being enrolled.

\section{Experimental task}

We used E-prime software (Schneider et al., 2002) to present stimuli and to record behavioural data, and all stimuli were presented in the centre of a $27-i n$. monitor with a resolution of $1,280 \times 720$ pixels over a grey background $(\mathrm{RGB}=180,180$, 180). The participants were comfortably seated in a quiet room with their head fixed on a chin rest approximately $60 \mathrm{~cm}$ from the centre of the monitor. Two temporal expectation tasks were performed by participants: a rhythmic cue task and a symbolic cue task, which were each performed separately, and the initial task was counter-balanced across participants. Both written and verbal instructions for the rhythmic and symbolic cue tasks (which are included in Fig. 1) were provided to the participants.

In both tasks, each trial started with the presentation of the fixation point for $500 \mathrm{~ms}$, which consisted of a black ' + ' symbol $\left(0.6^{\circ} \times 0.6^{\circ}\right.$ of visual angle), followed by the presentation of a rhythmic or a symbolic cue (Fig. 1). The rhythmic cue consisted of an isochronous sequence of stimuli consisting of four or five successive circles (at a uniform probability that was randomly generated during the trial), which appeared for $100 \mathrm{~ms}$ and disappeared every 500 or $1,500 \mathrm{~ms}$; the time interval changed randomly from trial to trial (depending on the interval condition; see Table 1). All stimuli were solid grey circles $\left(\right.$ diameter $\left.=1.2^{\circ} ; \mathrm{RGB}=100,100,100\right)$, except the final stimulus, which was a solid white circle $\left(\right.$ diameter $\left.=1.2^{\circ}\right)$ and served as the signal of an impending target that delivered the onset point of the following, preparatory, foreperiod (i.e., the interval between the cue and the target). This variation in the number of stimulus presentations prevented the white circle from being fully predictable. However, the symbolic cue was a digital presentation of ' 500 ' or ' 1,500 ' (Courier New 18 


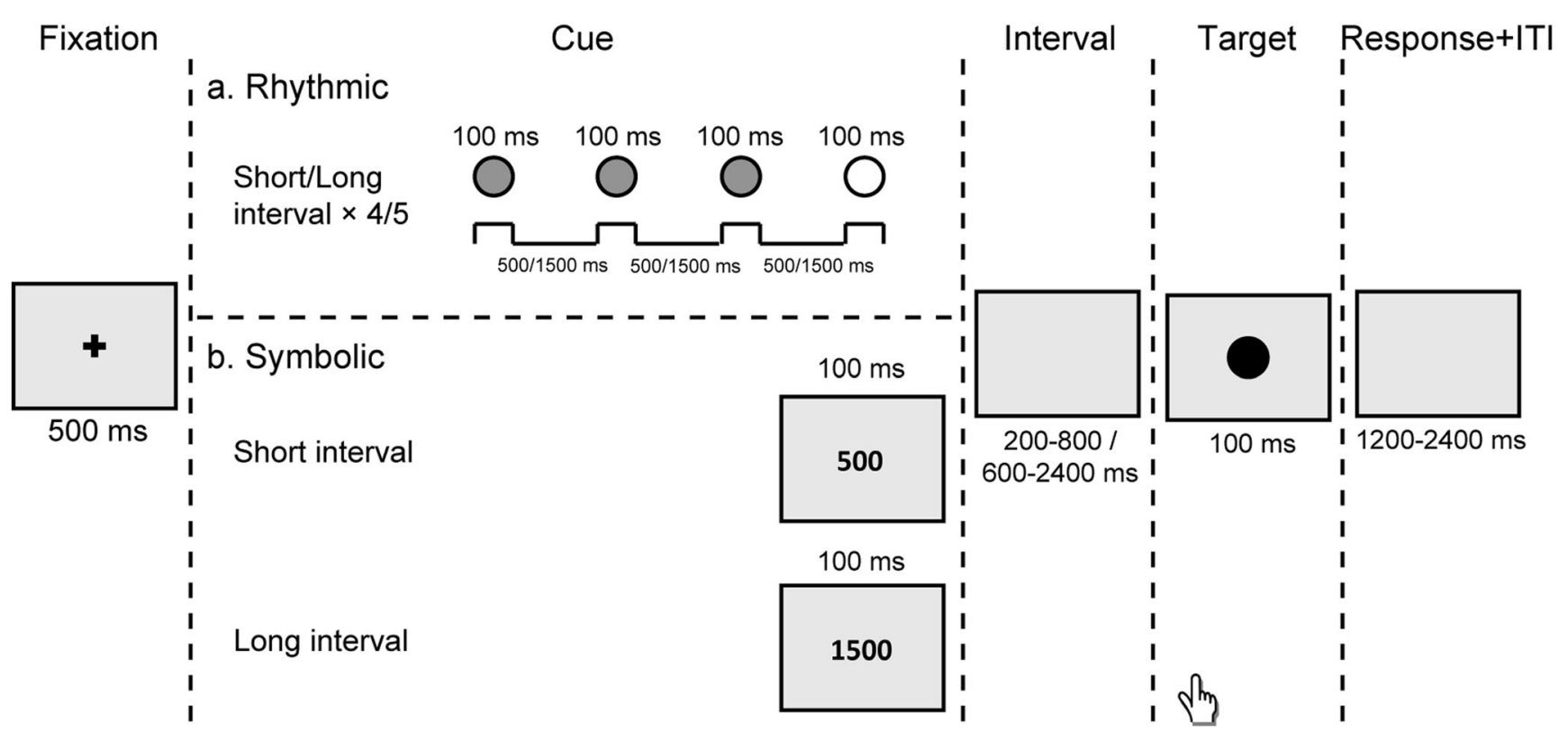

Fig. 1 Experimental task. Each trial started with the presentation of the fixation point for $500 \mathrm{~ms}$ followed by the presentation of a rhythmic or a symbolic cue. (a) The rhythmic cue consisted of four or five regular circle stimuli that appeared for $100 \mathrm{~ms}$ and disappeared every 500 or $1,500 \mathrm{~ms}$. (b) The symbolic cue was a digital visual ' 500 ' or ' 1,500 ' stimulus

point) with a duration of $100 \mathrm{~ms}$ that randomly appeared following the preceding fixation point. The digital presentation of ' 500 ' indicated that the target would appear after $500 \mathrm{~ms}$, whereas the digital presentation of ' 1,500 ' indicated that the target would appear after 1,500 ms. Following both temporal cues, a black circle target $\left(\right.$ diameter $=1.2^{\circ}$ ) appeared for $100 \mathrm{~ms}$ after a variable interval (described below; see Table 1); then, the screen remained blank until the participant responded or for 1,200 ms. The participants were instructed to respond as quickly as possible after the presentation of the target by pressing the left button with their index fingers while avoiding anticipation. The inter-trial interval was $1,200 \mathrm{~ms}$.

Both the rhythmic and symbolic cue tasks included 440 trials, which were equally distributed in 220 short-interval trials and 220 long-interval trials. The probability that the target appeared at the expected interval (i.e., $500 \mathrm{~ms}$ or $1,500 \mathrm{~ms}$ ) was set to $54 \%$ (240 trials). In the other $36 \%$ of trials (160 trials), the target appeared at an unexpected interval, and these times were distributed across four earlier

Table 1 Summary of all the cue-target intervals (ms) for the short- and long-interval conditions

\begin{tabular}{lllllllllll}
\hline & Interval & \multicolumn{6}{c}{ Temporal expectation (proportion of expected interval) } \\
\cline { 2 - 9 } & 0.4 & 0.55 & 0.7 & 0.85 & 1 & 1.15 & 1.3 & 1.45 & 1.6 \\
\hline Short & 200 & 275 & 350 & 425 & 500 & 575 & 650 & 725 & 800 \\
Long & 600 & 825 & 1,050 & 1,275 & 1,500 & 1,725 & 1,950 & 2,175 & 2,400 \\
\hline
\end{tabular}

presented for $100 \mathrm{~ms}$. Following either temporal cue, a black circle target appeared for $100 \mathrm{~ms}$ after a variable interval. The participants were instructed to respond as quickly as possible once the target appeared by pressing the left button with their index fingers while avoiding anticipation

intervals (at a proportion of $0.4,0.55,0.7$, or 0.85 of the expected interval) and four later intervals (at a proportion of $1.15,1.3,1.45$, or 1.6 of the expected interval). See Table 1, for the short-interval conditions, for which the expected interval was $500 \mathrm{~ms}$ and the unexpected interval was 200 , $275,350,425,575,650,725$ or $800 \mathrm{~ms}$. For the longinterval conditions, the expected interval was $1,500 \mathrm{~ms}$ and the unexpected interval was $600,825,1,050,1,275$, $1,725,1,950,2,175$ or $2,400 \mathrm{~ms}$. In $10 \%$ of the total trials (40 trials), the target was not presented (catch trials); this manipulation was included to prevent the target from being confidently expected at longer intervals, thus helping attenuate the effects of the 'hazard function', which is defined as the increasing conditional probability over time that the target is going to appear if it has not already occurred (Correa, Lupianez, \& Tudela, 2006; Nobrel, Correa, \& Coull, 2007). At the beginning of the experiment, the participants were instructed about the task, the catch trials and the final white circle in the rhythmic sequence that marked the beginning of the foreperiod preparatory interval. The participants were also encouraged to use the pace of the rhythmic or the symbolic cue to prepare for upcoming targets but to respond only when the target actually appeared while avoiding anticipation. Prior to each symbolic cue task, all the participants performed a training session with 24 trials. In the training phase, the foreperiod was always expected $(500 \mathrm{~ms}$ or $1,500 \mathrm{~ms}$ in two different blocks) to ensure a stable reference memory, thus allowing the participants to learn the association between the digital cues and 
Table 2 List of fixed-effect predictors

\begin{tabular}{lll}
\hline Predictor & Type & Levels (variants) \\
\hline Task & Categorical & Rhythmic Cue, Symbolic Cue \\
Interval & Categorical & Short, Long \\
Temporal expectation $\left(\mathrm{TE}, \mathrm{TE}^{2}\right)$ & Continuous, Quadratic polynomial & N/A \\
\hline
\end{tabular}

the foreperiod to establish a temporal expectation about the point in time of target onset.

\section{Data analysis}

The RT in both tasks refers to the time between the onset of the target and the motor response. The practice trials and catch trials were eliminated from the analyses. Anticipated responses (participants responding before the target appeared) and omission errors (participants did not respond after the target appeared) were excluded from the analysis (1.92\% of trials rejected). For each participant, trials were also discarded if the RT was larger than three standard deviations from the mean RT, which was separately calculated for each dependent variable (task, interval and temporal expectation) or if the RT was smaller than $50 \mathrm{~ms}$ (1.6\% rejected). The remaining correct mean RTs were modelled as a function of a number of variables, and mixed-effects linear regression analyses (Baayen, Davidson, \& Bates, 2008; Jc \& Bates, 2000) were conducted using the lmer function from the lme4 package (Bates et al. 2015) in R (vision 4.0.2). The set of fixed-effect predictors is summarized in Table 2 , and their interactions are included in all models.

Previous studies (Griffin, Miniussi, \& Nobre, 2001; Piras \& Coull, 2011) have reported some nonlinear effects of temporal expectation (TE) with a U-shaped temporal expectation

Table 3 Summary of all the fixed effect coefficients for the models of reaction time: coefficient estimates, standard errors, t-values and significance levels

\begin{tabular}{|c|c|c|c|c|}
\hline & Estimate & SE & $T$ & $P$ \\
\hline (Intercept) & 290.27 & 9 & 32.25 & $<0.001$ \\
\hline Task & 21.99 & 1.55 & 14.22 & $<0.001$ \\
\hline Interval & 30.32 & 1.55 & 19.6 & $<0.001$ \\
\hline $\mathrm{TE}$ & -20.82 & 2.58 & -8.08 & $<0.001$ \\
\hline $\mathrm{TE}^{2}$ & 186.39 & 5.8 & 32.16 & $<0.001$ \\
\hline Task $\times$ TE & 0.47 & 5.15 & 0.09 & 0.928 \\
\hline Task $\times \mathrm{TE}^{2}$ & -160.17 & 11.59 & -13.82 & $<0.001$ \\
\hline Interval $\times \mathrm{TE}$ & 32.3 & 5.15 & 6.27 & $<0.001$ \\
\hline Interval $\times \mathrm{TE}^{2}$ & 18.12 & 11.59 & 1.56 & 0.118 \\
\hline Task $\times$ Interval & -23.31 & 3.09 & -7.54 & $<0.001$ \\
\hline Task $\times$ Interval $\times \mathrm{TE}$ & 11.01 & 10.31 & 1.07 & 0.285 \\
\hline Task $\times$ Interval $\times \mathrm{TE}^{2}$ & -40.54 & 23.19 & -1.75 & 0.08 \\
\hline
\end{tabular}

curve; thus, a quadratic term $\left(\mathrm{TE}^{2}\right)$ was also included to model potential nonlinear effects in our data. TE were centred to minimize collinearity. For Task and Interval, simple coding (Rhythmic Cue: -.5; Symbolic Cue: .5; Short-Interval: -.5; Long-Interval: .5) was used.

To examine whether the temporal expectation curve differed by task and interval, the model also included a twoway interaction of temporal expectation $\left(\mathrm{TE}+\mathrm{TE}^{2}\right)$ with task and interval. The interaction between task and interval was also included to capture how the contrast between the two tasks differed by interval. The models also included a threeway interaction of temporal expectation $\left(\mathrm{TE}+\mathrm{TE}^{2}\right)$, task and interval to examine whether and how the interaction of temporal expectation and task varied by interval. The model included by-participant random intercepts as well as all possible random slopes to allow for variability among participants. The correlations between random effects were omitted to facilitate model convergence.

\section{Results}

The fixed effects for the statistical models of RT are summarized in Table 3; each fixed effect coefficient is shown with its standard error, test statistic and significance level, which were calculated using the Satterthwaite approximation as implemented in the ImerTest package (Kuznetsova, Brockhoff, \& Christensen, 2015).

First, compared with the symbolic cue task, the main effect of task is significant $(\beta=21.99, p<0.001)$ with a faster $\mathrm{RT}$ for the rhythmic cue task. A significant main effect of interval $(\beta$ $=30.32, p<0.001)$ is also observed, which shows that the RT for the short-interval condition was faster than that for the long-interval condition. Temporal expectation (TE) $(\beta=$ $20.82, p<0.001)$ and the quadratic term $\left(\mathrm{TE}^{2}\right)(\beta=186.39$, $p<0.001)$ are confirmed to be significant and well suited to capturing the effect of temporal expectation, with slower RTs for the earlier and later unexpected intervals and faster RTs for intervals approaching the expected point in time, which are apparent as U-shaped RT curves in Fig. 2.

Additionally, the interaction between Task $\times \mathrm{TE}^{2}$ is significant $(\beta=-160.17, p<0.001)$, indicating that the U-shaped RT curve for the rhythmic cue task is steeper than that for the symbolic cue task. The interaction between Task $\times$ Interval is also significant $(\beta=-23.31, p<0.001)$, showing that the RT 
for the rhythmic cue task is faster than that for the symbolic cue task during the short-interval conditions; however, during the long-interval condition, the RT for the symbolic cue task is faster. The Interval $\times$ TE interaction was also revealed to be significant $(\beta=32.3, p<0.001)$, where the difference of the RT between the short- and long-interval increases over the temporal expectation. The three-way interactions of Task $\times$ Interval $\times$ TE $(\beta=11.01, p=0.285)$ and Task $\times$ Interval $\times$ $\mathrm{TE}^{2}(\beta=-40.54, p=0.08)$ were not significant.

\section{Discussion}

The aim of the present experiments was to investigate the attentional precision of temporal expectations driven by rhythmic versus symbolic cues. By directly comparing rhythmic and symbolic cue tasks, the results showed a temporal expectation effect for both tasks: slower RTs for the earlier and later unexpected intervals and faster RTs for intervals approaching the expected point in time. Moreover, we found a significant interaction between task and the quadratic term of temporal expectation, indicating that the U-shaped RT curve for the rhythmic cue task is

(a)
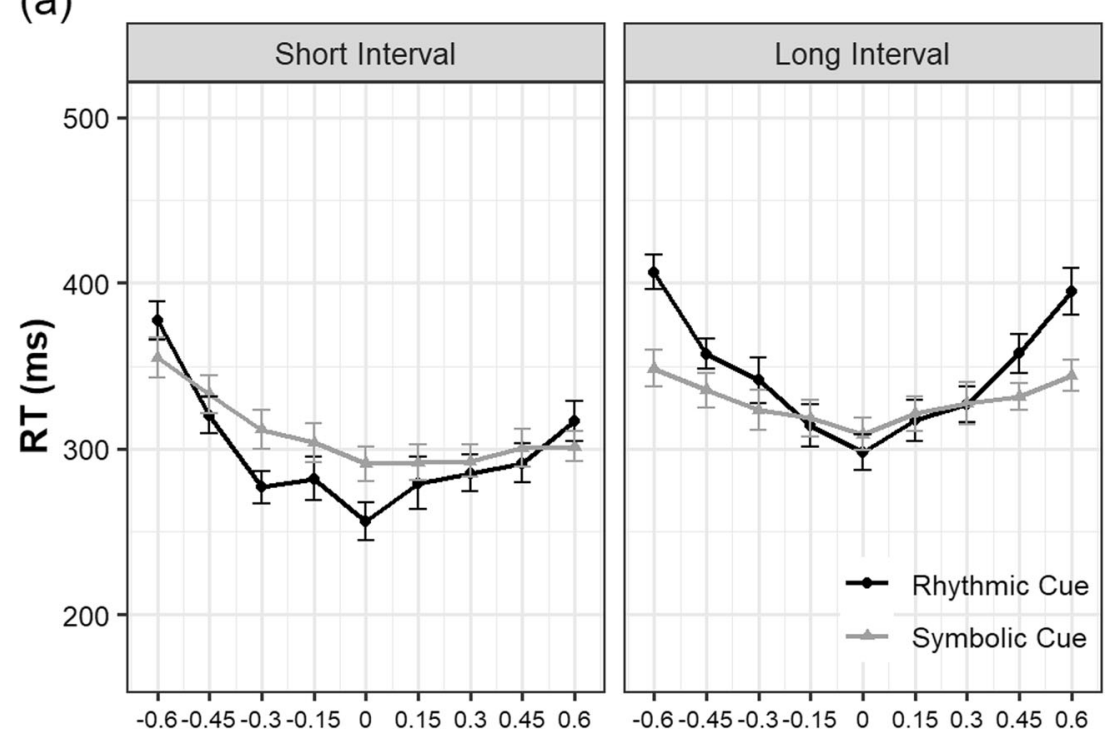

Temporal expectation

(b)

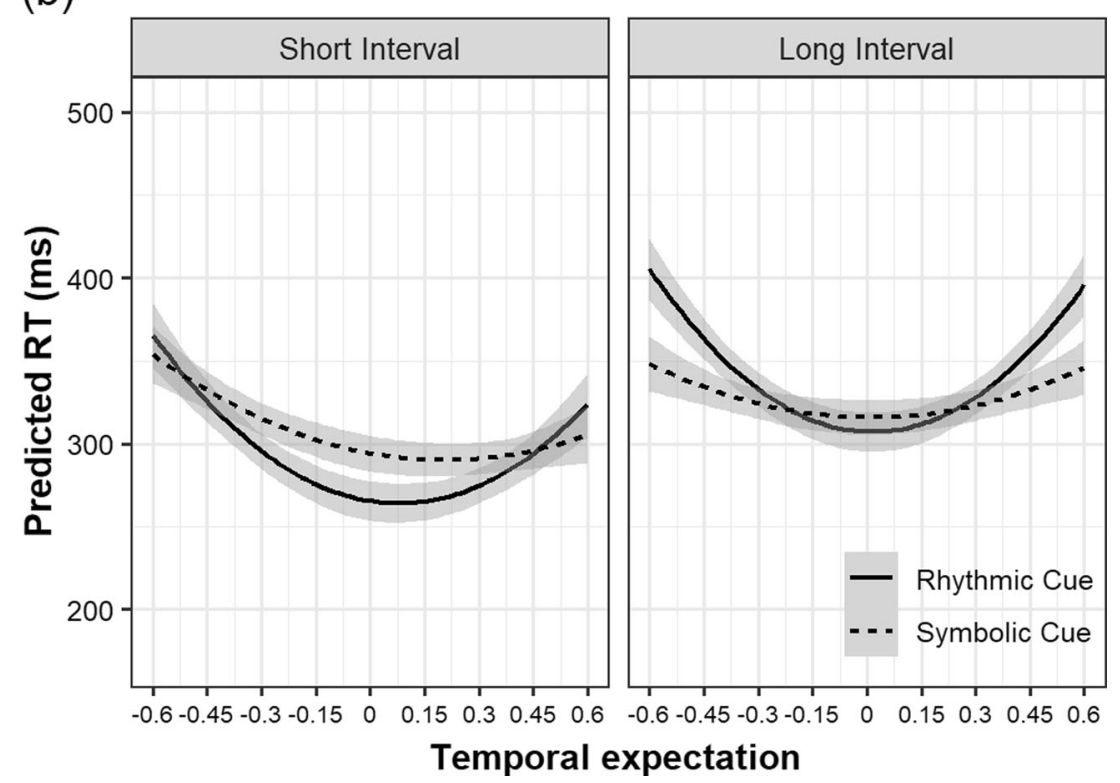

Fig. 2 Actual plots (a) and model prediction plots (b) for the reaction times (RTs) of the rhythmic and symbolic cue tasks for the short- and long-interval conditions as a function of temporal expectation. The $95 \%$ confidence intervals are shown in grey shading 
steeper than that for the symbolic cue task. This result revealed that compared with symbolic cues, temporal expectation driven by rhythmic cues provides a more precise attentional focus in time.

The responses to targets that appeared at the expected interval were observed to be faster in the rhythmic cue task than in the symbolic cue task under the short-interval conditions. However, no differences were observed under the longinterval conditions (see Fig. 2). A possible conjecture for this phenomenon was that a relatively short, constant foreperiod allows accurate and confident prediction of the target onset time, whereas a longer, constant interval results in predictions that are more variable and less accurate with higher uncertainty, hence resulting in a slower RT (Gibbon, Church, \& Meck, 1984). Moreover, a previous study found that when participants were asked to continue isochronous sequences by tapping, the variability in these sequences was minimal around an interval of $600 \mathrm{~ms}$; the variability increased for shorter or longer intervals (Fraisse, 1956). Furthermore, if the next stimulus lies too far beyond $2 \mathrm{~s}$ or $3 \mathrm{~s}$, then it becomes impossible to synchronize a regular sequence precisely, and the two stimuli are suggested to no longer to be perceptually linked: the rhythm disappears and the two stimuli appear as independent events (Fraisse, 1982; Mates, Müller, Radil, \& Pöppel, 1994; Poppel, 1997). Thus, as the prediction becomes less accurate during long intervals, the difference between the performance at the expected interval in the rhythmic and symbolic cue tasks was lessened.

U-shaped RT curves were found in both the rhythmic and symbolic cue tasks with a faster response to the target appearing around the expected relative to the unexpected intervals. However, under the short-interval conditions, we observed asymmetrical curves in both the rhythmic and symbolic cue tasks. These data indicated that although catch trials were included to prevent the effects of the 'hazard function', it was clear that the participants still used the temporal probability information inherent in the elapse of time itself to reorient their attention to the later interval if the target did not appear at the expected interval. Thus, we suggest that catch trials could only modulate the temporal probabilities and could not completely inhibit the hazard function. Consistent with our suggestion, previous studies that included $10 \%$ and $20 \%$ catch trials have found that the early and late unexpected targets were processed differently (Correa \& Nobre, 2008; Jones, Hsu, Granjon, \& Waszak, 2017).

Our important result is that the U-shaped RT curves for the rhythmic cue task are steeper than those for the symbolic cue task. However, previous studies have observed U-shaped RT curves in both rhythmic (Jones et al., 2017; Sanabria et al., 2011) and symbolic cue tasks (Griffin et al., 2001). Nonetheless, the curves for the two forms of temporal expectation have rarely been compared directly. Our current study provides novel evidence that the U-shaped RT curves for the rhythmic cue task are steeper than those for the symbolic cue task, revealing that rhythmic cues allowed the participants to allocate more focal attention around the cued expected point in time. One conceivable interpretation of this result may be that temporal expectations driven by symbolic cues are based on a remembered standard code in the reference memory (Church, 1984; John Gibbon, 1977; Treisman, 1963). However, temporal expectation, as driven by the relative timing of onsets of successive symbolic cues, has been related to the self-sustaining entrainment model (Jones \& Boltz, 1989; Large \& Jones, 1999). Thus, the isochronous sequence afforded a more robust, stable and accurate temporal expectation against a single memorized interval.

\section{Conclusion}

The results of this study showed a significant interaction between task and the quadratic term of temporal expectation, indicating that the U-shaped RT curve for the rhythmic cue task is steeper than that for the symbolic cue task. These outcomes revealed that compared with symbolic cues, temporal expectation driven by rhythmic cues provides a more precise attentional focus in time.

Acknowledgements This study was supported by the Japan Society for the Promotion of Science (JSPS) KAKENHI grant numbers 16K18052, 17K18855, 18H05009, 18K12149 and 18H01411, a Grant-in-Aid for Strategic Research Promotion from Okayama University, the National Natural Science Foundation of China (31800932), Humanity and Social Science Youth Foundation of the Ministry of Education of China (18XJC190003), Innovation and Entrepreneurship project for Highlevel Overseas Talent of Guizhou Province [(2019)04], and Doctor Scientific Research Staring Foundation of the Guizhou University of Traditional Chinese Medicine (2018[21). We are grateful to Otsuka Toshimi Scholarship Foundation for providing support. We would like to express our gratitude to all the subjects who participated in our study.

\section{References}

Baayen, R Harald, Davidson, Douglas J, \& Bates, Douglas M. (2008). Mixed-effects modeling with crossed random effects for subjects and items. Journal of memory and language, 59(4), 390-412.

Barnes, R., \& Jones, M. R. (2000). Expectancy, attention, and time. Cognitive Psychology, 41(3), 254-311. https://doi.org/10.1006/ cogp. 2000.0738

Bates, D, Maecher, M, Bolker, B, Walker, S, Christensen, RHB, \& Singmann, H. (2015). lme4: Linear mixedeffects models using Eigen and S4. R package, version 1.1-10. In.

Breska, A., \& Deouell, L. Y. (2014). Automatic Bias of Temporal Expectations following Temporally Regular Input Independently of High-level Temporal Expectation. Journal of Cognitive Neuroscience, 26(7), 1555-1571. https://doi.org/10.1162/jocn a 00564

Capizzi, M., Sanabria, D., \& Correa, A. (2012). Dissociating controlled from automatic processing in temporal preparation. Cognition, 123(2), 293-302. https://doi.org/10.1016/j.cognition.2012.02.005 
Church, R. M. (1984). Properties of the internal clock. Ann N Y Acad Sci, 423, 566-582.

Correa, A., Cona, G., Arbula, S., Vallesi, A., \& Bisiacchi, P. (2014). Neural dissociation of automatic and controlled temporal preparation by transcranial magnetic stimulation. Neuropsychologia, 65 , 131-136. https://doi.org/10.1016/j.neuropsychologia.2014.10.023

Correa, A., Lupianez, J., Milliken, B., \& Tudela, P. (2004). Endogenous temporal orienting of attention in detection and discrimination tasks. Percept Psychophys, 66(2), 264-278.

Correa, A., Lupianez, J., \& Tudela, P. (2006). The attentional mechanism of temporal orienting: determinants and attributes. Exp Brain Res, 169(1), 58-68. https://doi.org/10.1007/s00221-005-0131-x

Correa, A., \& Nobre, A. C. (2008). Neural modulation by regularity and passage of time. Journal of Neurophysiology, 100(3), 1649-1655. https://doi.org/10.1152/jn.90656.2008

Coull, J. T., Frith, C. D., Buchel, C., \& Nobre, A. C. (2000). Orienting attention in time: behavioural and neuroanatomical distinction between exogenous and endogenous shifts. Neuropsychologia, 38(6), 808-819. https://doi.org/10.1016/S0028-3932(99)00132-3

Coull, J. T., \& Nobre, A. C. (1998). Where and when to pay attention: the neural systems for directing attention to spatial locations and to time intervals as revealed by both PET and fMRI. $J$ Neurosci, 18(18), 7426-7435.

Doherty, J. R., Rao, A., Mesulam, M. M., \& Nobre, A. C. (2005). Synergistic effect of combined temporal and spatial expectations on visual attention. $J$ Neurosci, 25(36), 8259-8266. https://doi.org/ 10.1523/JNEUROSCI.1821-05.2005

Fraisse, Paul. (1956). Les structures rythmiques : étude psychologique. Louvain: Publications universitaires de Louvain.

Fraisse, Paul. (1982). Rhythm and tempo. The psychology of music, 1, 149-180.

Gibbon, J., Church, R. M., \& Meck, W. H. (1984). Scalar timing in memory. Ann N Y Acad Sci, 423, 52-77.

Gibbon, John. (1977). Scalar expectancy theory and Weber's law in animal timing. Psychol Rev, 84(3), 279.

Griffin, I. C., Miniussi, C., \& Nobre, A. C. (2001). Orienting attention in time. Frontiers in Bioscience, 6, D660-D671. https://doi.org/10. 2741/Griffin

Jc, Pinheiro, \& Bates, DM. (2000). Mixed-effects models in S and SPLUS. Springer, New York.

Jones, A., Hsu, Y. F., Granjon, L., \& Waszak, F. (2017). Temporal expectancies driven by self- and externally generated rhythms. Neuroimage, 156, 352-362. https://doi.org/10.1016/j.neuroimage. 2017.05.042

Jones, M. R., \& Boltz, M. (1989). Dynamic attending and responses to time. Psychol Rev, 96(3), 459-491.

Jones, M. R., Moynihan, H., MacKenzie, N., \& Puente, J. (2002). Temporal aspects of stimulus-driven attending in dynamic arrays.
Psychol Sci, 13(4), 313-319. https://doi.org/10.1111/1467-9280. 00458

Kuznetsova, A., Brockhoff, P., \& Christensen, R. (2015). LmerTest: Tests in linear mixed effects models. $R$ Package Version, 2. https://doi.org/10.18637/jss.v082.i13

Large, E. W., \& Jones, M. R. (1999). The dynamics of attending: How people track time-varying events. Psychol Rev, 106(1), 119-159. https://doi.org/10.1037/0033-295x.106.1.119

Mates, Jiří, Müller, Ulrike, Radil, Tomáš, \& Pöppel, Ernst. (1994). Temporal integration in sensorimotor synchronization. Journal of Cognitive Neuroscience, 6(4), 332-340.

McAuley, J. D., \& Jones, M. R. (2003). Modeling effects of rhythmic context on perceived duration: a comparison of interval and entrainment approaches to short-interval timing. J Exp Psychol Hum Percept Perform, 29(6), 1102-1125. https://doi.org/10.1037/00961523.29.6.1102

Nobrel, A. C., Correa, A., \& Coull, J. T. (2007). The hazards of time. Current Opinion in Neurobiology, 17(4), 465-470. https://doi.org/ 10.1016/j.conb.2007.07.006

Piras, F., \& Coull, J. T. (2011). Implicit, Predictive Timing Draws upon the Same Scalar Representation of Time as Explicit Timing. Plos One, 6(3). ARTN e18203. https://doi.org/10.1371/journal.pone. 0018203

Poppel, E. (1997). A hierarchical model of temporal perception. Trends Cogn Sci, 1(2), 56-61. https://doi.org/10.1016/S1364-6613(97) 01008-5

Rohenkohl, G., Coull, J. T., \& Nobre, A. C. (2011). Behavioural Dissociation between Exogenous and Endogenous Temporal Orienting of Attention. Plos One, 6(1). ARTN e14620. https://doi. org/10.1371/journal.pone.0014620

Sanabria, D., Capizzi, M., \& Correa, A. (2011). Rhythms That Speed You Up. Journal of Experimental Psychology-Human Perception and Performance, 37(1), 236-244. https://doi.org/10.1037/ a0019956

Schneider, Walter, Eschman, Amy, \& Zuccolotto, Anthony. (2002). EPrime: User's guide: Psychology Software Incorporated.

Treisman, Michel. (1963). Temporal discrimination and the indifference interval: implications for a model of the "internal clock.". Washington: American Psychological Association.

Trivino, M., Arnedo, M., Lupianez, J., Chirivella, J., \& Correa, A. (2011). Rhythms can overcome temporal orienting deficit after right frontal damage. Neuropsychologia, 49(14), 3917-3930. https://doi.org/10. 1016/j.neuropsychologia.2011.10.009

Publisher's note Springer Nature remains neutral with regard to jurisdictional claims in published maps and institutional affiliations. 